\title{
Renaissance of leptin for obesity therapy
}

\author{
Carmelo Quarta $^{1}$ - Miguel A. Sánchez-Garrido ${ }^{1}$ - Matthias H. Tschöp ${ }^{1,2,3}$ • \\ Christoffer Clemmensen ${ }^{1}$
}

Received: 4 December 2015 / Accepted: 22 January 2016 /Published online: 16 March 2016

(C) Springer-Verlag Berlin Heidelberg 2016

\begin{abstract}
Diet-induced obesity and its metabolic comorbidities constitute an overwhelming health crisis and there is an urgent need for safe and effective pharmacological interventions. Being largely shelved for decades, scientists are now revisiting the antiobesity virtues of leptin. Whereas it remains evident that leptin as a stand-alone therapy is not an effective approach, the potential for employing sensitising pharmacology to unleash the weight-lowering properties of leptin has injected new hope into the field. Fascinatingly, these leptin-sensitising agents seem to act via distinct metabolic pathways and may thus, in parallel with their clinical development, serve as important research tools to progress our understanding of the molecular, physiological and behavioural pathways underlying energy homeostasis and obesity pathophysiology. This review summarises a presentation given at the 'Is leptin coming back?' symposium at the 2015 annual meeting of the EASD. It is accompanied by two other reviews on topics from this symposium (by Thomas Meek and Gregory Morton, DOI: 10.1007/s00125-016-3898-3, and by Gerald Shulman and colleagues, DOI: 10.1007/s00125016-3909-4) and an overview by the Session Chair, Ulf Smith (DOI: 10.1007/s00125-016-3894-7).
\end{abstract}

Carmelo Quarta and Miguel A. Sánchez-Garrido contributed equally to this paper.

Matthias H. Tschöp

tschoep@helmholtz-muenchen.de

1 Institute for Diabetes and Obesity, Helmholtz Diabetes Center, Helmholtz Zentrum München, Ingolstädter Landstraße 1, 85764 Neuherberg, Munich, Germany

2 Division of Metabolic Diseases, Department of Medicine, Technische Universität München, Munich, Germany

3 German Center for Diabetes Research (DZD), Neuherberg, Munich, Germany
Keywords Leptin - Leptin resistance $\cdot$ Leptin sensitivity · Obesity $\cdot$ Pharmacology $\cdot$ Polypharmacy $\cdot$ Review $\cdot$ Type 2 diabetes
Abbreviations
DIO Diet-induced obese
ER Endoplasmic reticulum
FGF21 Fibroblast growth factor 21
GLP-1 Glucagon-like peptide 1
HSF1 Heat shock transcription factor 1
IKK $\beta \quad$ IкB kinase $\beta$
MyD88 Myeloid differentiation primary response gene 88
PGC1 $\alpha$ Peroxisome proliferator-activated receptor- $\gamma$ coactivator $1 \alpha$
POMC Pro-opiomelanocortin
PTP1B Protein tyrosine phosphatase 1B
SOCS3 Suppressor of cytokine signalling 3
STAT3 Signal transducer and activator of transcription 3
TLR4 Toll-like receptor 4

\section{Introduction}

Although the misperception of obesity as a consequence of poor self-control and gluttony is still widespread, body weight has a strong genetic component orchestrating the neuroendocrine systems that govern food intake and energy expenditure [1]. When the adipocyte-derived hormone leptin and its profound impact on central circuits controlling energy and glucose metabolism were discovered [2], hopes were raised that obesity might be able to be reversed with single-hormone therapy. Discouragingly, most obese individuals are hyperleptinaemic and exogenous leptin has a negligible effect on suppressing appetite to lower body weight [3]. This insight gave rise to 
the hypothesis of 'leptin resistance' as a phenomenon that may play a crucial role in the proximate perturbations in energy homeostasis that ultimately lead to obesity [4]. However, whether or not the inability of leptin to suppress feeding in the face of obesity is a consequence of cellular 'leptin resistance' remains subject to debate and is beyond the scope of this current review. Irrespective of this dispute, amplifying leptin responsiveness may hold the key to unlocking the potential of leptin as a powerful anti-obesity drug.

Whereas it is well established that leptin can be used to correct neuroendocrine and metabolic abnormalities in patients with acquired or congenital leptin deficiency and lipodystrophy [5-7], overcoming resistance to the effects of leptin therapy on energy metabolism in common obesity continues to challenge us. Intriguingly, in recent years, novel approaches have surfaced to show that leptin resistance is a reversible condition. Indeed, preclinical studies have identified several 'leptin sensitisers', that may hold therapeutic promise. These leptin sensitisers target distinct neuroendocrine systems, all of which have been linked to leptin signalling pathology, including endoplasmic reticulum (ER) stress [8], hypothalamic inflammation [9] and multiple nodes of the leptin signalling cascade [10-13]. In addition, a growing body of evidence suggests that gastrointestinalderived peptides and a series of already approved pharmacotherapies can amplify the weight-lowering actions of exogenous leptin [14-19], re-positioning leptin as a relevant agent in combination therapies for the treatment of obesity.

In this review we present an overview of emerging strategies for restoring or enhancing the energy metabolic actions of leptin in the leptin-resistant state. We summarise the proposed cellular and molecular underpinnings of these novel leptinenhancing precision medicines and we provide an outlook on the translational prospects and challenges and, accordingly, the rational for employing leptin in future polypharmacy to treat common obesity.

\section{Targeting leptin responsiveness with pharmacology}

Whether the inadequacy of hyperleptinaemia to correct obesity reflects a diet-induced defect in leptin action or whether the predominant physiological role of leptin is to defend against reductions in body fat remains a topic of discussion and ongoing investigations [20, 21]. Separately from this debate, a growing body of evidence supports the thinking that distinct pharmacological interventions can act as applicable leptin sensitisers in the obese leptin-resistant state (see Text box). This notion of pharmacotherapy-induced 'turbocharging' of leptin signalling is contributing to a paradigm shift, positioning leptin as a possible adjunctive agent in future polypharmacological anti-obesity interventions. In the following sections, the most promising strategies to enhance leptin responsiveness in obesity are reviewed.
ER stress The ER is a dynamic organelle regulating the synthesis, folding and maturation of proteins. An imbalance between the loading and folding capacities of the ER results in a condition known as ER stress. Pioneering studies have demonstrated a causal link between hypothalamic ER stress, leptin signalling and obesity [22, 23] (Fig. 1). Corroborating this theory, preclinical studies found that specific overexpression of molecular ER components can prevent dietary inhibition of leptin signalling [22]. Accordingly, pharmacological application of the chemical chaperones tauroursodeoxycholic acid (TUDCA) and 4-phenyl butyric acid (4-PBA) can alleviate hypothalamic ER stress to reverse diet-induced leptin resistance [22-24]. These chemical chaperones stabilise protein folding by decreasing the formation of abnormal protein aggregates, which reduces ER stress. In support of the hypothesis that ER stress may be directly involved in diet-induced leptin resistance and obesity progression, the non-steroidal anti-inflammatory drug (NSAID) flurbiprofen was recently shown to alleviate ER stress, and lower body weight in dietinduced obese (DIO) mice $[25,26]$.

The beneficial effects of the chemical chaperones in rodents suggest that they may enhance leptin action in obese individuals [27]. TUDCA was recently tested in obese insulin-resistant patients in a 4-week treatment study [28]. Although the treatment induced moderate improvements in insulin sensitivity, it exhibited no ability to lower body weight. Discouragingly, ER stress markers did not appear to be affected in peripheral tissues, and the relative weak capacity of these chaperones to improve ER function [29] may limit their clinical utility.

An in silico screening to identify novel small molecules acting on ER stress was recently performed [8]. This study led to the identification of a small molecule (celastrol) with potent anorectic and body weight-lowering effects in DIO mice. Importantly, compared with celastrol monotherapy, cotreatment of celastrol and leptin amplified weight loss in leptin resistant DIO mice, indicative of celastrol-induced restoration of leptin action. Supporting the interplay between celastrol administration and leptin, in obese mice with genetically disrupted leptin signalling ( $o b / o b$ and $d b / d b$ mice), celastrol treatment showed negligible effects on energy metabolism. While the effects of celastrol to correct hyperleptinaemia and obesity coincided with changes in hypothalamic STAT3 phosphorylation and a reduction in ER stress markers, a more recent study showed that celastrol induces an HSF1-PGC1 $\alpha$ axis to control thermogenic and mitochondrial gene programs in adipocytes and myocytes [30]. Independent of the primary site of action of celastrol, future investigations will undoubtedly explore safety and translational efficacy of celastrol and/ or celastrol mimetics to treat obesity.

Inflammation Diet-induced obesity promotes chronic lowgrade inflammation in both peripheral tissues and in the brain 


\begin{tabular}{|c|c|c|c|}
\hline \multicolumn{4}{|c|}{ Pharmacological leptin sensitisers } \\
\hline Compound & Metabolic effects & Mechanism of action & Species \\
\hline 4-PBA & $\begin{array}{l}\text { FI \& BW } \downarrow \\
\text { Insulin sensitivity } \uparrow\end{array}$ & $\begin{array}{l}\text { ER stress } \downarrow \\
\text { p-PERK } \downarrow\end{array}$ & Mouse \\
\hline TUDCA & $\begin{array}{l}\text { FI \& BW } \downarrow \\
\text { Insulin sensitivity } \uparrow\end{array}$ & $\begin{array}{l}\text { ER stress } \downarrow \\
\text { p-PERK } \downarrow\end{array}$ & Mouse \\
\hline Flurbiprofen & BW \& adiposity $\downarrow$ & $\begin{array}{l}\text { ER stress } \downarrow \\
\text { Protein aggregation/ inflammation } \downarrow\end{array}$ & Mouse \\
\hline Trodusquemine & FI, BW \& adiposity $\downarrow$ & $\begin{array}{l}\text { LepR signalling } \uparrow \\
\text { PTP1B } \downarrow\end{array}$ & Mouse \\
\hline Celastrol & $\begin{array}{l}\text { FI \& BW } \downarrow \\
\mathrm{EE} \uparrow \\
\text { Glucose tolerance, insulin sensitivity } \uparrow\end{array}$ & $\begin{array}{l}\text { ER stress } \downarrow \\
\text { p-PERK, SERCA2B } \downarrow \\
\text { HSF1-PGC1 } \uparrow \uparrow\end{array}$ & Mouse \\
\hline Amylin & FI, BW \& adiposity $\downarrow$ & $\begin{array}{l}\text { LepR signalling } \uparrow \\
\text { p-STAT3 } \uparrow\end{array}$ & $\begin{array}{l}\text { Rat and } \\
\text { human }\end{array}$ \\
\hline Exendin-4 & $\begin{array}{l}\text { FI, BW \& adiposity } \downarrow \\
\text { Glucose tolerance } \uparrow\end{array}$ & Exogenous leptin action $\uparrow$ & Mouse \\
\hline FGF21 & $\begin{array}{l}\text { BW \& adiposity } \downarrow \\
\text { Glucose tolerance } \uparrow\end{array}$ & Exogenous leptin action $\uparrow$ & Mouse \\
\hline GLP-1/Glucagon & $\begin{array}{l}\text { FI, BW and adiposity } \downarrow \\
\text { Glucose tolerance } \uparrow\end{array}$ & Exogenous leptin action $\uparrow$ & Mouse \\
\hline Clusterin & FI \& BW $\downarrow$ & $\begin{array}{l}\text { Exogenous leptin action } \uparrow \\
\text { LepR signalling } \uparrow \\
\text { LepR binding } \uparrow\end{array}$ & Mouse \\
\hline Metformin & $\begin{array}{l}\text { FI, BW \& adiposity } \downarrow \\
\text { Insulin sensitivity } \uparrow\end{array}$ & $\begin{array}{l}\text { Exogenous leptin action } \uparrow \\
\text { LepR signalling } \uparrow \\
\text { Leptin transport across the BBB } \uparrow\end{array}$ & Rat \\
\hline $\mathrm{mCPP}$ & $\mathrm{BW} \downarrow$ & $\begin{array}{l}\text { Exogenous leptin action } \uparrow \\
\text { LepR signalling } \uparrow \\
\text { p-STAT3 } \uparrow\end{array}$ & Mouse \\
\hline PYY(3-36) & FI \& BW $\downarrow$ & Exogenous leptin action $\uparrow$ & Rat \\
\hline CCK & FI $\downarrow$ & LepR signalling $\uparrow$ & Mouse \\
\hline
\end{tabular}

BBB, blood-brain barrier; BW, body weight; CCK, cholecystokinin; EE, energy expenditure; ER, endoplasmic reticulum; FGF21, fibroblast growth factor 21; FI, food intake; GLP-1, glucagon-like peptide-1; HSF1, heat shock transcription factor 1; LepR, leptin receptor; mCPP, metachlorophenylpiperazine; PBA, 4-phenyl butyric acid; PERK, protein kinase RNA-like endoplasmic reticulum kinase; PGC1 $\alpha$, peroxisome proliferator-activated receptor-gamma coactivator 1alpha; PTP1B, protein tyrosine phosphatase 1B; PYY, peptide YY; PVN, paraventricular nucleus of the hypothalamus; SERCA2B, sarcoplasmic/endoplasmic reticulum calcium ATPase 2; STAT3, signal transducer and activator of transcription 3; TUDCA, tauroursodeoxycholic acid

[31]. In the hypothalamus, the amplified immune response to excess nutrients may play a causal role for pathogenesis of diet-induced leptin resistance and adiposity [32, 33] (Fig. 1). Excess dietary fat activates TLR4-MyD88-dependent $\mathrm{IKK} \beta / \mathrm{NF}-\mathrm{KB}$ signalling, which in turn leads to the production and release of proinflammatory cytokines to impair hypothalamic leptin signalling [24, 34, 35]. Moreover, diet- induced induction in proinflammatory cytokines triggers an immediate response in non-neuronal hypothalamic cells (microglial and astroglial populations) [33, 36, 37]. Corroborating a role for proinflammatory cytokines in leptin signalling pathology, central administration of TNF- $\alpha$ impairs leptin sensitivity, possibly by increasing the levels of SOCS3 and PTP1B, which act as negative regulators of 


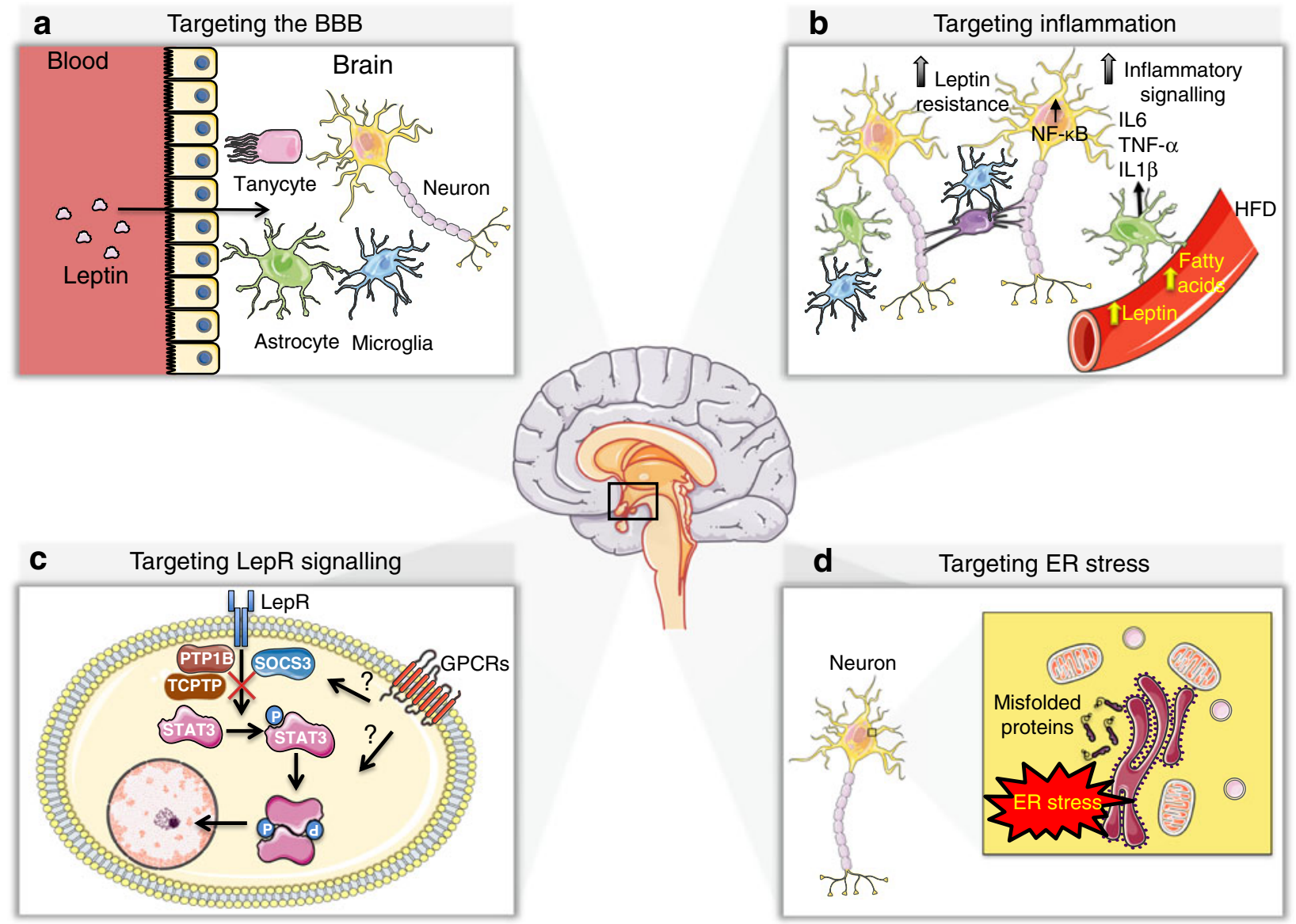

Fig. 1 Schematic overview of potential therapeutic strategies to enhance leptin action. (a) Agents that facilitate increased leptin transport across the blood-brain barrier and/or tanycytes may provide enhanced leptin access to hypothalamic target cells. (b) Targeting hypothalamic inflammation in neurons or glial cells may reverse diet-induced perturbations in cytokine signalling and NF- $\mathrm{KB}$ signalling to restore leptin sensitivity. (c) Negative regulators of leptin receptor (LepR) intracellular signalling, including the protein tyrosine phosphatases PTP1B, TCPTP and SOCS3 may be relevant targets to increase the maximal signalling capacity downstream of

leptin receptor signalling $[9,11,38]$. Although causality is not clear, there is considerable evidence to support the existence of a vicious cycle between excess nutrients, hypothalamic inflammation, leptin resistance and obesity [39]. Of note, glial leptin receptor signalling was recently reported to control feeding [40]. However, the relative contribution of astrocyte leptin signalling to diet-induced leptin resistance, hypothalamic inflammation and obesity is to date uncharted.

Several studies have explored the potential of pharmacological intervention targeting hypothalamic IKK $\beta / \mathrm{NF}-\mathrm{kB}$ signalling to reverse diet-induced metabolic perturbations. Teasaponin, a naturally derived inhibitor of NF- $\mathrm{kB}$, has been reported to reduce body weight and improve leptin sensitivity in DIO mice [41]. IKK $\beta / \mathrm{NF}-\mathrm{kB}$ inhibitors have been found to reduce food intake and lower body weight via increasing leptin sensitivity in some, but not all studies, indicating that
LepR. Multiple G protein-coupled receptors (GPCRs) are intertwined with leptin signalling. Indeed, peripheral administration of several approved GPCR-targeting pharmacotherapies can enhance leptin responsiveness in leptin-resistant obesity. (d) Correction of imbalances in neuronal protein folding, i.e. ER stress, via application of chemical chaperones, might increase central leptin sensitivity and restore energy homeostasis. HFD, high-fat diet; TCPTP, T Cell PTP. The figure was produced using Servier Medical Art (www.servier.com)

the application of this strategy needs further evaluation [32, 42-45].

Future studies investigating the mechanistic interplay between diet-induced molecular perturbations in glial cells, hypothalamic inflammation-like processes and the development of leptin resistance are urgently needed. Furthermore, the role of neuronal-supporting cell types such as tanycytes in this pathology, e.g. through controlling leptin entry across the blood-brain barrier, deserves additional attention (Fig. 1). Insights into these cell type-specific processes and their interconnectedness could potentially facilitate the discovery of novel drug candidates that reverse leptin resistance.

Metformin Treatment with the glucose-lowering drug metformin disproportionally decreases circulating leptin levels in obese and healthy individuals [46-48] and, in DIO rats, reverses leptin resistance [16]. In addition to restoring leptin 
responsiveness in DIO leptin-resistant rats, metformin treatment also enhances the anorectic effect of exogenous leptin in lean rats. The efficacy of metformin to restore leptin sensitivity may be multi-factorial and involve enhanced transport of leptin across the blood-brain barrier, increased leptin receptor expression and/or induction of the STAT3-POMC signalling pathway [16, 49]. Furthermore, metformin directly inhibits leptin secretion in rat adipocytes [50]. Studies in obese leptin-resistant humans are needed to evaluate whether metformin is able to restore the weight-lowering activity of exogenous leptin and, thus, if metformin and leptin co-treatment can reduce body weight synergistically and should be considered as a relevant anti-obesity combinatorial strategy. Of note, despite the fact that metformin has been widely used as a type 2 diabetes therapy for many years, its mechanisms of action remain poorly defined. Uncovering the cellular metabolic actions of metformin may, in parallel, provide important hints for its application as a leptin-sensitising agent.

Amylin A landmark study by Roth and colleagues demonstrated that pretreating obese humans or rodents with the pancreatic polypeptide amylin restored the responsiveness to exogenous leptin in terms of weight-lowering [14]. In DIO leptin-resistant rats, co-administration of leptin and amylin led to a greater body weight loss than monotherapy. Importantly, the efficacy of amylin in restoring the weightlowering efficacy of leptin was confirmed in obese and overweight patients subjected to energy restriction and pramlintide (amylin analogue) and metreleptin (leptin analogue) as combination therapy [51, 52]. At the molecular level, amylin was shown to restore leptin sensitivity by potentiating leptinstimulated phosphorylation of STAT3 in the arcuate nucleus and the ventromedial hypothalamus (VMH). Additionally, complementary neuronal signalling in the area postrema, and the mesolimbic reward pathway between amylin and leptin has been reported $[53,54]$. Although only mild and transient adverse effects were reported with the combination of pramlintide and metreleptin, in 2011, Amylin Inc. decided to halt the development of this combination therapy because of potential safety issues. Whether this relates to metreleptininduced generation of antibodies with neutralising activity remains speculative, however, in the meantime, metreleptin has been approved for treating leptin deficiency in patients with congenital or acquired lipodystrophy [55-57].

\section{Glucagon-like peptide 1 and fibroblast growth factor 21 In} 2012, Müller and colleagues reported that pharmacologically induced restoration of leptin responsiveness is not a feature unique to amylin, but that fibroblast growth factor 21 (FGF21) and exendin-4 (glucagon-like peptide 1 [GLP-1] receptor agonist) can also restore sensitivity to the catabolic actions of exogenous leptin when co-administered [15]. In this study, a site-specific PEGylated leptin was used to enhance bioavailability relative to native leptin. Although FGF21 and exendin-4 act via distinct metabolic pathways, the efficacy of exogenous leptin to amplify the pharmacologically induced weight loss was, for both compounds, not achieved until $\sim 25 \%$ weight loss had occurred, suggesting a threshold for the restoration of leptin sensitivity. One caveat to FGF21and exendin-4-mediated restoration of leptin responsiveness was the necessity of a concomitant dietary switch to a low-fat diet upon treatment initiation. Strikingly, energy restrictioninduced weight loss to $\sim 25 \%$ alone did not restore leptin responsiveness, suggesting that both weight loss pharmacotherapy and leptin receptor activation are required to restore the metabolic action profile of leptin (Fig. 1).

Corroborating the differential effect between pharmacologically-mediated and energy restriction-mediated weight loss, a recent study demonstrated that GLP-1R agonism, but not energy restriction, drives a reduction in nutrient-induced hypothalamic inflammation [36]. However, further investigations are required to establish whether the ability of GLP-1R agonism to amplify leptin sensitivity is causally linked to a reversal in diet-induced microgliosis.

GLP-1 receptor/glucagon receptor co-agonism Recent progress in biotechnology has helped facilitate the generation of a series of single-molecule compounds that combine and integrate different modes of pharmacological action [58]. In 2009 , this strategy was used to show that a molecule with balanced action at the glucagon receptor and the GLP-1 receptor (GLP-1R), synergistically orchestrates distinct biological pathways to exhibit a coordinated effect on energy metabolism in rodents [59]. This GLP-1R/glucagon receptor coagonist was recently found to act as a powerful leptin sensitiser in leptin-resistant DIO mice [60]. Indeed, animals who received co-treatment with GLP-1/glucagon and leptin exhibited superior weight loss relative to each monotherapy. Importantly, and in contrast to treatment with exendin-4 and FGF21 [15], the GLP-1R/glucagon receptor co-agonist restored leptin responsiveness in DIO mice chronically exposed to a high-fat, high sucrose diet. While it remains to be uncovered how exactly the dual agonist activating glucagon- and GLP-1 receptors alleviates leptin unresponsiveness, the antiobesity properties of concerted GLP-1R and glucagon receptor co-agonism translates to humans [61, 62], and several pharmaceutical companies are now pursuing GLP/glucagon co-agonism for the treatment of obesity [58].

\section{Conclusions and future directions}

While the discovery of leptin as a governing anorectic signal did not immediately lead to the development of an influential anti-obesity drug, it triggered massive interest in delineating the neuronal circuitries underling the central control of energy 
homeostasis. Some 20 years later, there is a much deeper understanding of the neuronal machinery involved in the control of feeding behaviour and systemic substrate metabolism. However, despite major parallel advances in biotechnology, synthetic chemistry and information technology, these efforts have yet to be translated into safe and efficacious anti-obesity pharmacotherapies.

One way of overcoming such frustrating complexity of energy homeostasis regulation may be offered by simultaneous targeting of several distinct biological mechanisms. Specifically, novel poly-agonism molecules may offer unprecedented potential to overcome severe diet-induced energy metabolic perturbations and offset redundant counterregulatory processes. Importantly, as outlined in this review, leptin may serve a key role in next-generation anti-obesity polypharmacy. While major efforts continue to be invested in understanding and refining ways of re-sensitising the neuronal circuitry to the weight-lowering properties of leptin, focus on the feasibility of targeting these pathways with translational pharmacology should be pursued with equal determination.

Engineering mixed small and large molecule co-agonists could represent another viable strategy to specifically deliver abundant concentrations of leptin sensitisers into hypothalamic target cells. We published the first proof-of-concept showing that peptide hormones can be exploited to selectively target steroid hormones in a cell-specific manner governed by the peptide receptor expression pattern [63]. A stable GLP-1oestrogen conjugate was thus developed to selectively deliver oestrogen to GLP-1R-expressing cell populations, including the hypothalamus. The specific targeting of oestrogen amplified the anorectic properties of each co-agonist constituent without the hallmark toxicities of oestrogen actions in nonGLP1R-expressing cells. This conceptual approach could be evolved to decorate GLP-1 or other macromolecules with leptin-sensitising small molecules targeting ER stress or inhibiting inflammation.

There is reason for optimism. Our knowledge on the molecular basis of leptin signalling has never been greater, and if we continue to work hard on translating emerging molecular insights into therapeutic strategies we may, in the near future, be able to sensitise leptin signalling in obese individuals. As one example for key areas warranting intense future studies, major efforts will be necessary to understand how cell types such as tanycytes, microglia and astrocytes interact with neurons to meaningfully integrate existing knowledge of leptin biology with neuroendocrine pathologies resulting from-and driving - metabolic disease. Importantly, for leptin to (re)enter clinical studies as anti-obesity therapy, previously reported adverse effects associated with leptin administration, including hypertension and immunogenicity, will have to be carefully assessed $[64,65]$.
Nevertheless, we may currently be witnessing a shift in the anti-obesity pharmacology paradigm. Recent biotechnological advancements have pushed the development of singlemolecule biologics with engineered mixed-agonism as well as next-generation small molecule screens resulting in the identification and validation of potent novel leptin sensitisers. By overcoming leptin insensitivity, such approaches appear to offer some of the most promising strategies yet to reverse and prevent diet-induced obesity, insulin resistance and type 2 diabetes.

Duality of interest The authors declare that there is no duality of interest associated with this manuscript.

Contribution statement All authors were responsible for drafting the article and for critically revising it for substantial intellectual content. All authors approved the publication of this article.

\section{References}

1. van der Klaauw AA, Farooqi IS (2015) The hunger genes: pathways to obesity. Cell 161:119-132

2. Zhang Y, Proenca R, Maffei M, Barone M, Leopold L, Friedman JM (1994) Positional cloning of the mouse obese gene and its human homologue. Nature 372:425-432

3. Heymsfield SB, Greenberg AS, Fujioka K et al (1999) Recombinant leptin for weight loss in obese and lean adults: a randomized, controlled, dose-escalation trial. JAMA 282:1568-1575

4. Myers MG Jr, Leibel RL, Seeley RJ, Schwartz MW (2010) Obesity and leptin resistance: distinguishing cause from effect. Trends Endocrinol Metab 21:643-651

5. Oral EA, Simha V, Ruiz E et al (2002) Leptin-replacement therapy for lipodystrophy. N Engl J Med 346:570-578

6. Farooqi IS, Jebb SA, Langmack G et al (1999) Effects of recombinant leptin therapy in a child with congenital leptin deficiency. N Engl J Med 341:879-884

7. Farooqi IS, Matarese G, Lord GM et al (2002) Beneficial effects of leptin on obesity, T cell hyporesponsiveness, and neuroendocrine/ metabolic dysfunction of human congenital leptin deficiency. J Clin Invest 110:1093-1103

8. Liu J, Lee J, Salazar Hernandez MA, Mazitschek R, Ozcan U (2015) Treatment of obesity with celastrol. Cell 161:999-1011

9. de Git KC, Adan RA (2015) Leptin resistance in diet-induced obesity: the role of hypothalamic inflammation. Obes Rev 16:207-224

10. Pedroso JA, Buonfiglio DC, Cardinali LI et al (2014) Inactivation of SOCS3 in leptin receptor-expressing cells protects mice from diet-induced insulin resistance but does not prevent obesity. Mol Metab 3:608-618

11. Picardi PK, Caricilli AM, de Abreu LL, Carvalheira JB, Velloso LA, Saad MJ (2010) Modulation of hypothalamic PTP1B in the TNF- $\alpha$-induced insulin and leptin resistance. FEBS Lett 584: 3179-3184

12. Lantz KA, Hart SG, Planey SL et al (2010) Inhibition of PTP1B by trodusquemine (MSI-1436) causes fat-specific weight loss in dietinduced obese mice. Obesity 18:1516-1523

13. Byun K, Gil SY, Namkoong C et al (2014) Clusterin/ApoJ enhances central leptin signaling through Lrp2-mediated endocytosis. EMBO Rep 15:801-808 
14. Roth JD, Roland BL, Cole RL et al (2008) Leptin responsiveness restored by amylin agonism in diet-induced obesity: evidence from nonclinical and clinical studies. Proc Natl Acad Sci U S A 105: $7257-7262$

15. Müller TD, Sullivan LM, Habegger K et al (2012) Restoration of leptin responsiveness in diet-induced obese mice using an optimized leptin analog in combination with exendin-4 or FGF21. J Pept Sci 18:383-393

16. Kim YW, Kim JY, Park YH et al (2006) Metformin restores leptin sensitivity in high-fat-fed obese rats with leptin resistance. Diabetes 55:716-724

17. Yan C, Yang Y, Saito K et al (2015) Meta-chlorophenylpiperazine enhances leptin sensitivity in diet-induced obese mice. Br J Pharmacol 172:3510-3521

18. Wang L, Barachina MD, Martinez V, Wei JY, Tache Y (2000) Synergistic interaction between CCK and leptin to regulate food intake. Regul Pept 92:79-85

19. Unniappan S, Kieffer TJ (2008) Leptin extends the anorectic effects of chronic PYY(3-36) administration in ad libitum-fed rats. Am J Physiol Regul Integr Comp Physiol 295:R51-R58

20. Ravussin Y, Leibel RL, Ferrante AW Jr (2014) A missing link in body weight homeostasis: the catabolic signal of the overfed state. Cell Metab 20:565-572

21. Ottaway N, Mahbod P, Rivero B et al (2015) Diet-induced obese mice retain endogenous leptin action. Cell Metab 21:877-882

22. Ozcan L, Ergin AS, Lu A et al (2009) Endoplasmic reticulum stress plays a central role in development of leptin resistance. Cell Metab 9:35-51

23. Cakir I, Cyr NE, Perello M et al (2013) Obesity induces hypothalamic endoplasmic reticulum stress and impairs proopiomelanocortin (POMC) post-translational processing. J Biol Chem 288:17675-17688

24. Zhang X, Zhang G, Zhang H, Karin M, Bai H, Cai D (2008) Hypothalamic IKK $\beta / \mathrm{NF}-\mathrm{kB}$ and ER stress link overnutrition to energy imbalance and obesity. Cell 135:61-73

25. Hosoi T, Baba S, Ozawa K (2014) Therapeutic potential of flurbiprofen against obesity in mice. Biochem Biophys Res Commun 449:132-134

26. Hosoi T, Yamaguchi R, Noji K et al (2014) Flurbiprofen ameliorated obesity by attenuating leptin resistance induced by endoplasmic reticulum stress. EMBO Mol Med 6:335-346

27. Henstridge DC, Whitham M, Febbraio MA (2014) Chaperoning to the metabolic party: the emerging therapeutic role of heat-shock proteins in obesity and type 2 diabetes. Mol Metab 3:781-793

28. Kars M, Yang L, Gregor MF et al (2010) Tauroursodeoxycholic acid may improve liver and muscle but not adipose tissue insulin sensitivity in obese men and women. Diabetes 59:1899-1905

29. Park SW, Ozcan U (2013) Potential for therapeutic manipulation of the UPR in disease. Semin Immunopathol 35:351-373

30. Ma X, Xu L, Alberobello AT et al (2015) Celastrol protects against obesity and metabolic dysfunction through activation of a HSF1PGC1 $\alpha$ transcriptional axis. Cell Metab 22:695-708

31. Gregor MF, Hotamisligil GS (2011) Inflammatory mechanisms in obesity. Annu Rev Immunol 29:415-445

32. Valdearcos M, Xu AW, Koliwad SK (2015) Hypothalamic inflammation in the control of metabolic function. Annu Rev Physiol 77: $131-160$

33. Thaler JP, Yi CX, Schur EA et al (2012) Obesity is associated with hypothalamic injury in rodents and humans. J Clin Invest 122:153-162

34. Kleinridders A, Schenten D, Konner AC et al (2009) MyD88 signaling in the CNS is required for development of fatty acid-induced leptin resistance and diet-induced obesity. Cell Metab 10:249-259

35. Milanski M, Degasperi G, Coope A et al (2009) Saturated fatty acids produce an inflammatory response predominantly through the activation of TLR4 signaling in hypothalamus: implications for the pathogenesis of obesity. J Neurosci 29:359-370
36. Gao Y, Ottaway N, Schriever SC et al (2014) Hormones and diet, but not body weight, control hypothalamic microglial activity. Glia $62: 17-25$

37. De Souza CT, Araujo EP, Bordin S et al (2005) Consumption of a fatrich diet activates a proinflammatory response and induces insulin resistance in the hypothalamus. Endocrinology 146:4192-4199

38. Zabolotny JM, Kim YB, Welsh LA, Kershaw EE, Neel BG, Kahn BB (2008) Protein-tyrosine phosphatase 1B expression is induced by inflammation in vivo. J Biol Chem 283:14230-14241

39. Thaler JP, Guyenet SJ, Dorfman MD, Wisse BE, Schwartz MW (2013) Hypothalamic inflammation: marker or mechanism of obesity pathogenesis? Diabetes 62:2629-2634

40. Kim JG, Suyama S, Koch M et al (2014) Leptin signaling in astrocytes regulates hypothalamic neuronal circuits and feeding. Nat Neurosci 17:908-910

41. Yu Y, Wu Y, Szabo A et al (2013) Teasaponin reduces inflammation and central leptin resistance in diet-induced obese male mice. Endocrinology 154:3130-3140

42. Posey KA, Clegg DJ, Printz RL et al (2009) Hypothalamic proinflammatory lipid accumulation, inflammation, and insulin resistance in rats fed a high-fat diet. Am J Physiol Endocrinol Metab 296:E1003-E1012

43. Jang PG, Namkoong C, Kang GM et al (2010) NF- $k B$ activation in hypothalamic pro-opiomelanocortin neurons is essential in illnessand leptin-induced anorexia. J Biol Chem 285:9706-9715

44. Oh IS, Thaler JP, Ogimoto K, Wisse BE, Morton GJ, Schwartz MW (2010) Central administration of interleukin-4 exacerbates hypothalamic inflammation and weight gain during high-fat feeding. Am J Physiol Endocrinol Metab 299:E47-E53

45. Benzler J, Ganjam GK, Pretz D et al (2015) Central inhibition of $\mathrm{IKK} \beta / \mathrm{NF}-\mathrm{kB}$ signaling attenuates high-fat diet-induced obesity and glucose intolerance. Diabetes 64:2015-2027

46. Kay JP, Alemzadeh R, Langley G, D'Angelo L, Smith P, Holshouser S (2001) Beneficial effects of metformin in normoglycemic morbidly obese adolescents. Metab Clin Exp 50:1457-1461

47. Glueck CJ, Fontaine RN, Wang P et al (2001) Metformin reduces weight, centripetal obesity, insulin, leptin, and low-density lipoprotein cholesterol in nondiabetic, morbidly obese subjects with body mass index greater than 30. Metab Clin Exp 50:856-861

48. Fruehwald-Schultes B, Oltmanns KM, Toschek B et al (2002) Short-term treatment with metformin decreases serum leptin concentration without affecting body weight and body fat content in normal-weight healthy men. Metab Clin Exp 51:531-536

49. Aubert G, Mansuy V, Voirol MJ, Pellerin L, Pralong FP (2011) The anorexigenic effects of metformin involve increases in hypothalamic leptin receptor expression. Metab Clin Exp 60:327-334

50. Mueller WM, Stanhope KL, Gregoire F, Evans JL, Havel PJ (2000) Effects of metformin and vanadium on leptin secretion from cultured rat adipocytes. Obes Res 8:530-539

51. Aronne L, Fujioka K, Aroda V et al (2007) Progressive reduction in body weight after treatment with the amylin analog pramlintide in obese subjects: a phase 2, randomized, placebo-controlled, doseescalation study. J Clin Endocrinol Metab 92:2977-2983

52. Ravussin E, Smith SR, Mitchell JA et al (2009) Enhanced weight loss with pramlintide/metreleptin: an integrated neurohormonal approach to obesity pharmacotherapy. Obesity 17:1736-1743

53. Mietlicki-Baase EG, Olivos DR, Jeffrey BA, Hayes MR (2015) Cooperative interaction between leptin and amylin signaling in the ventral tegmental area for the control of food intake. Am J Physiol Endocrinol Metab 308:E1116-E1122

54. Turek VF, Trevaskis JL, Levin BE et al (2010) Mechanisms of amylin/ leptin synergy in rodent models. Endocrinology 151:143-152

55. Sinha G (2014) Leptin therapy gains FDA approval. Nat Biotechnol 32:300-302

56. Meehan CA, Cochran E, Kassai A, Brown RJ, Gorden P (2016) Metreleptin for injection to treat the complications of leptin 
deficiency in patients with congenital or acquired generalized lipodystrophy. Expert Rev Clin Pharmacol 9:59-68

57. Rodriguez AJ, Mastronardi CA, Paz-Filho GJ (2015) New advances in the treatment of generalized lipodystrophy: role of metreleptin. Ther Clin Risk Manag 11:1391-1400

58. Finan B, Clemmensen C, Muller TD (2015) Emerging opportunities for the treatment of metabolic diseases: Glucagon-like peptide-1 based multi-agonists. Mol Cell Endocrinol 418P1: $42-54$

59. Day JW, Ottaway N, Patterson JT et al (2009) A new glucagon and GLP-1 co-agonist eliminates obesity in rodents. Nat Chem Biol 5: 749-757

60. Clemmensen C, Chabenne J, Finan B et al (2014) GLP-1/ glucagon coagonism restores leptin responsiveness in obese mice chronically maintained on an obesogenic diet. Diabetes 63:1422-1427
61. Cegla J, Troke RC, Jones B et al (2014) Coinfusion of low-dose GLP-1 and glucagon in man results in a reduction in food intake. Diabetes 63:3711-3720

62. Tan TM, Field BC, McCullough KA et al (2013) Coadministration of glucagon-like peptide-1 during glucagon infusion in humans results in increased energy expenditure and amelioration of hyperglycemia. Diabetes 62:1131-1138

63. Finan B, Yang B, Ottaway N et al (2012) Targeted estrogen delivery reverses the metabolic syndrome. Nat Med 18:1847-1856

64. Simonds SE, Cowley MA (2013) Hypertension in obesity: is leptin the culprit? Trends Neurosci 36:121-132

65. Chan JL, Koda J, Heilig JS et al (2015) Immunogenicity associated with metreleptin treatment in patients with obesity or lipodystrophy. Clin Endocrinol. doi:10.1111/cen.12980 